\title{
REVISITANDO A INTEROPERABILIDADE NO CONTEXTO DOS ACERVOS DIGITAIS
}

\author{
REVISITING INTEROPERABILITY IN THE CONTEXT \\ OF DIGITAL ACCOUNTS
}

Jose Eduardo Santarem Segundo ${ }^{1}$
Marcel Ferrante Silva
Dalton Lopes Martins ${ }^{3}$

\section{RESUMO}

A interoperabilidade é a capacidade de sistemas distintos trocarem dados entre si, independente de plataformas, hardware ou software. Apesar de a interoperabilidade ser debatida há bastante tempo, ela ainda é um entrave nos processos de integração de dados de acervos digitais, dificultando os processos de agregação e acesso único a uma variedade de fontes diversas. 0 objetivo desta pesquisa é identificar e apresentar as possibilidades de interoperabilidade técnica e de conteúdo inerentes a ambientes e plataformas que implementam acervos digitais. A metodologia utilizada baseia-se principalmente em levantamento bibliográfico sobre "acervos digitais" e "interoperabilidade". Como resultado foi possível compreender que ainda há uma grande dificuldade em interoperar dados oriundos de fontes diferentes, e que melhorar os conhecimentos sobre as possibilidades de uso dos recursos de interoperabilidade pode ser imprescindível para que equipes possam efetivar a integração de dados de acervos digitais diversos.

Palavras-chave: Interoperabilidade. Interoperabilidade técnica. Mapeamento da interoperabilidade. Acervos digitais.

\begin{abstract}
Interoperability is the ability of disparate systems to exchange data between each other, regardless of platform, hardware, or software. Although interoperability is being debated for a long time, it is still a hindrance to the processes of data integration of digital collections, making it difficult to aggregate processes and access only to a variety of different sources. The objective of this research is to identify and present the possibilities of technical and content interoperability inherent to environments and platforms that implement digital collections. The methodology used is mainly based on a bibliographic survey on "digital collections" and "interoperability". As a result, it was possible to understand that there is still great difficulty in interoperating data from different sources, and that improving knowledge about the possibilities of using the interoperability resources may be essential for teams to be able to effectively integrate the data of diverse digital collections.
\end{abstract}

Keywords: Interoperability.Technical interoperability.Interoperability Mapping.Digital collections.

Artigo recebido em 9/02/2018 e aceito para publicação em 12/03/2018.

1 Doutor em Ciência da Informação pela Universidade Estadual Paulista Júlio de Mesquita Filho, Brasil. Docente permanente do Programa de Pós-Graduação em Ciência da Informação da Universidade Estadual Paulista Julio de Mesquita Filho, Brasil. E-mail: santarem@usp.br.

2 Doutor em Ciência da Informação pela Universidade Federal de Minas Gerais, Brasil. Professor da Universidade Federal de Goiás, Brasil. E-mail: marcelf@gmail.com.

3 Doutor em Ciência da Informação pela Universidade de São Paulo, Brasil. Professor da Universidade de Brasília, Brasil. E-mail: dmartins@gmail.com. 


\section{INTRODUÇÃO}

Esta pesquisa aborda questões sobre interoperabilidade, apesar de o termo estar constantemente em uso e já ter sido discutido em muitos trabalhos de pesquisa, mostra-se cada vez mais atual a necessidade de se rediscutir e pautar como requisito necessário para evolução e revolução dos acervos digitais. Serviços e produtos disponibilizados em projetos e políticas culturais demandam soluções que ampliam seu valor de uso em função de maior capacidade de abrangência. Também do lado da difusão do valor dos serviços o desafio da interoperabilidade aparece.

Sempre, neste texto, que o termo acervo digital for encontrado deve-se entender como uma forma abrangente de expressar todo tipo de plataforma digital que compreenda dados e/ou documentos, ou seja, biblioteca, repositório, museu e arquivo corrente, intermediário ou permanente. Portanto outros termos mais específicos como bibliotecas e repositórios digitais, museus digitais e/ou virtuais também podem ser utilizados em exemplos ou citações, mas reforça-se a ideia de pensar em acervos de uma maneira abrangente e global.

0 contexto atual tem na informação e no conhecimento os requisitos chave para contribuir com a evolução da humanidade. Entretanto, a grande quantidade de tecnologias recentes, a produção constante de conteúdo, as redes sociais, o paradigma do big data, o uso massivo das nuvens, as humanidades digitais e todos os processos de conversão de material analógico em digital, tem tornado latente a necessidade de minimizar o desafio da humanidade em acessar e localizar conteúdos de forma mais eficiente. A integração torna-se necessidade nos processos indispensáveis para transformar informação em conhecimento de maior alcance.

A realidade atual nos apresenta uma imensidão de dados, ou ainda, grandes conjuntos de dados, mas que estão dispostos de forma isolada, sem ligação entre eles, e principalmente com dificuldade de consumo pela comunidade que precisa desses dados.

Fusco $(2010$, p.76) diz que

A complexidade do compartilhamento de informações foi ampliada nos últimos anos com a utilização de ambientes altamente distribuídos e heterogêneos como a web e a busca por normas, padrões, regras e protocolos que permitam uma organização e representação da informação de modo que se possa alcançar a interoperabilidade.

Este estudo debruça-se, especificamente, sobre o contexto dos acervos digitais e seus processos de troca de informações, entendendo que 0 acesso a conteúdos digitais é complementar aos meios tradicionais de acesso a conteúdo analógico, apesar de ganhar cada vez mais espaço e importância 
como estratégia de socialização da informação, nos mais variados segmentos de informação e conhecimento. Os acervos digitais tornaram-se meio de acesso a informação na saúde, na educação, na economia e principalmente como oportunidade de acesso a cultura, entre outras áreas. Entende-se que as diferentes estratégias de agregação produzidas por iniciativas de interoperabilidade agregam valor não apenas por permitir que os dados estejam disponíveis para acesso em espaço, mas também por permitirem se encontrar padrões e tendências que apenas são percebidas quando a escala da análise se amplia.

Entende-se, portanto, como fortemente estratégica a democratização do acesso por meio dos acervos digitais, potencializando a difusão do conhecimento que outrora ficava restrito à visitação e acessos físicos in loco. 0 acesso, por meio dos acervos digitais, fomenta a promoção do conhecimento, da cultura e principalmente da capacidade de geração de ideias e inovação e aceleração do desenvolvimento.

Ross (2003, p.5) identificou esses aspectos ao caracterizar uma biblioteca digital como “infra-estrutura, políticas e procedimentos, e mecanismos organizacionais, políticos e econômicos necessários para permitir o acesso e a preservação do conteúdo digital”.

Esta pesquisa parte do pressuposto que que iniciativas como Europeana, Google Arts\&Culture, DigitalNZ, DPLA, World Digital Library, TROVE, Internet Archive entre outras plataformas similares, tem alterado a maneira de se coletar e disseminar conteúdo, mais especificamente cultural, no âmbito do mundo digital. Entender essas novas maneiras é passo necessário para a constituição de serviços abrangentes e interoperáveis dos acervos brasileiros, independente de sua área de conhecimento.

Permitir o acesso ao conteúdo por meio de acervos digitais, não passa apenas pela simples disponibilização dos mesmos, há um grande conjunto de atividades técnicas que precisam ser realizadas para que seja possível atender as necessidades das comunidades que terão acesso a esses conteúdos.

Martins e Silva (2017, p. 101) afirmam que:

\begin{abstract}
Um elemento estratégico de grande importância na concepção de uma biblioteca digital encontra-se na necessidade de projetar funcionalidades, formas de interação, modos de organização da informação e funcionamento do fluxo informacional que atenda às expectativas tanto dos gestores desse serviço quanto de sua potencial comunidade de usuários. Torna-se, portanto, um desafio entender como traduzir as demandas de gestão e funcionamento do serviço em funcionalidades técnicas que devem ser atendidas por um sistema de informação customizado para tal.
\end{abstract}

Disponibilizar à comunidade um acervo digital, portanto, é um processo composto por um grande conjunto de tarefas, dentre elas destacam-se: a seleção do conteúdo do acervo; caracterização 
do seu modelo de funcionamento (agregador de índice, biblioteca digital); organização para seleção das origens/fontes de conteúdo; processo de escolha de um software (plataforma) com suas devidas funcionalidades e configurações; definição do melhor modelo de interface para o usuário e seus mais variados dispositivos de acesso e de ajustes/configuração da plataforma para integração com outros ambientes digitais.

Algumas destas tarefas: a escolha de um software, o modelo de funcionamento e a integração com outros ambientes digitais estão totalmente interligadas, e é necessária uma perfeita sintonia para que 0 acervo projetado possa atender as expectativas iniciais que justificaram 0 desenvolvimento do projeto. É de fundamental importância que o produto final seja compatível com o que foi pensado no início pelos idealizadores. Essas três tarefas têm a interoperabilidade como elemento comum e fundamental de funcionamento.

A cenário atual dos acervos digitais nos mostra uma grande quantidade de softwares (plataformas), alguns open source, que são utilizados em maior escala, parte deles proprietários e ainda uma pequena parte de soluções caseiras.Os conteúdos, objetos digitais, são oriundos de uma diversidade grande de ambientes (bibliotecas, museus, arquivos e outros) e precisam de integração para que haja relação entre eles, necessitam, para isso, de modelos claros e funcionais de interoperabilidade.

Entre bibliotecas digitais, o conceito de integração e interoperabilidade consiste na possibilidade de um usuário realizar buscas a recursos informacionais heterogêneos, armazenados em diferentes servidores na rede, utilizando-se de uma interface única sem tomar conhecimento de onde nem como estes recursos estão armazenados (MARCONDES; SAYÃO, 2002).

Esta pesquisa apresenta especificamente um estudo sobre o tema da interoperabilidade no contexto dos acervos digitais.

Algumas perguntas de pesquisa que norteiam este estudo são: quais são os protocolos disponíveis para interoperabilidade em plataformas de acervos digitais? Quais são os modos de funcionamento desses protocolos? Quais são as possibilidades de interoperabilidade entre as plataformas que implementam acervos digitais?

0 objetivo principal desta pesquisa é identificare apresentar as possibilidades de interoperabilidade técnica e de conteúdo inerentes a ambientes e plataformas que implementam acervos digitais. Como objetivos específicos apresentam-se um levantamento bibliográfico sobre o tema da interoperabilidade e a identificação e mapeamento dos principais modelos de interoperabilidade em uso entre as plataformas mais utilizadas para implementação de acervos digitais. Ao final, também destacam-se 
algumas inovações que foram percebidas pela equipe de pesquisa ao longo de seus estudos. Mesmo ainda sendo timidamente incorporadas pela produção científica da área, podem representar avanços significativos nos modelos de interoperabilidade atualmente existentes.

Espera-se que os resultados aqui apresentados possam contribuir no processo de interoperabilidade afim de prover integração entre conteúdos com formatos diferentes, oriundo de ambientes e fontes diversas e nas mais variadas plataformas e sistemas disponíveis e em uso atualmente, incluindo ferramentas abertas e proprietárias.

\section{PROCEDIMENTOS METODOLÓGICOS}

A natureza desta pesquisa é qualitativa, do tipo descritiva-exploratória. Busca analisar as possibilidades de modelos de interoperabilidade para uso em plataformas que implementam acervos digitais. Para tanto, inicia-se com uma abordagem sobre o conceito de interoperabilidade e posteriormente discorre sobre as possibilidades de interoperabilidade, propondo um modelo de mapeamento baseado na literatura disponível. A literatura consultada foi selecionada inicialmente nas bases Web of Science (WOS) e LISA: Library and Information Science Abstracts (LISA). Como o termo interoperabilidade é bastante utilizado, optou-se por um recorte temporal a partir do ano de 2010, utilizando-se apenas os resultados mais freqüentes até 0 ano de 2017.

As chaves de busca utilizadas nas duas bases de dados foram baseadas nos seguintes termos: "interoperability and digital library", "interoperability and repository" e "semantic interoperability and digital library".

Apesar do recorte temporal, foram recuperados 914 resultados na base LISA e 279 na WOS, e uma casa de intersecção de aproximadamente 10 a 15\% nos resultados das três chaves de busca utilizadas.

Um primeiro olhar sobre os resultados originados pelas duas bases identificou facilmente que 0 conteúdo de interesse para essa pesquisa estava muito mais presente na base LISA do que nos artigos selecionados na base WOS, entretanto optou-se por não descartar os resultados da base WOS.

Entendendo que nosso objeto são os acervos digitais e suas questões de interoperabilidade, iniciou-se o processo de identificar os artigos que pudessem ter em seu objeto essa temática.

Assim, chegou-se a um corpus de aproximadamente 250 artigos que tem em seu núcleo fundamental de estudo a questão da interoperabilidade em acervos digitais. 
0 objetivo inicial da busca era encontrar novas propostas e soluções de interoperabilidade que pudessem garantir alguma inovação no tema. Entretanto, quase que a totalidade dos textos abordavam questões relacionadas a problemas e soluções (já universalmente conhecidas) para interoperar dados. 0 principal destaque nesses artigos selecionados é a incapacidade dos sistemas de operarem entre si, a dificuldade das equipes em criar complementos de software para efetivar interoperabilidade e, ainda, o uso inadequado e irresponsável dos padrões de metadados. Diante do exposto, apresenta-se na próxima seção uma amostra dessa realidade.

\section{ESTADO DA ARTE}

Menzies, Birrell e Dunsire (2011) apresentam uma pesquisa que investigou a questão da interoperabilidade entre os catálogos online de acesso público (OPACs) e repositórios digitais nas universidades do Reino Unido. 0 projeto combinou métodos de pesquisa quantitativa e qualitativa, incluindo um questionário on-line distribuído ao pessoal em 85 universidades, levantamento bibliográfico e dois estudos de caso realizados nas Universidades de Cambridge e Glasgow.

Apenas $2 \%$ responderam afirmativamente quando perguntados se seus sistemas compartilhavam e processavam diretamente os dados uns dos outros. Foram encontrados 20 padrões de metadados diferentes e 12 padrões de conteúdo diferentes em uso em 85 Repositórios Institucionais. No entanto, pouca evidência de alinhamento de políticas ou de mapeamento e trabalho para melhorar a gama de correspondência de tipos de sistema, recursos e usuários, apesar de essas práticas terem sido defendidas por organismos de padrões por mais de uma década.

A interoperabilidade não está posicionada como uma prioridade clara e a maioria dos sistemas que não são de bibliotecas ainda não são considerados partes significativas (ou potencialmente significativas) da infraestrutura das Bibliotecas Digitais. Em geral, o compartilhamento de dados é abordado em uma base ad hoc ou experimental, principalmente para auxiliar na coleta de dados para outros frameworks de pesquisa.

Potvin e Thompson (2016) discutem a questão das teses e dissertações em formato eletrônico (ETDs) e a relação de importância dos metadados e da interoperabilidade. Realizaram uma abordagem identificando o design de vários padrões de metadados, oferecendo um estudo de caso focado nos esforços da Biblioteca Digital do Texas para forjar um padrão comum para ETDs. Intensificam a pesquisa na questão relacionada a inconsistência de metadados e, principalmente, às falhas de interoperabilidade geradas principalmente por filosofias divergentes sobre o papel dos metadados. 
Os autores indicam claramente que as instituições seguem um padrão de metadados para ETD's para atender a Networked Digital Library of Theses and Dissertations (NDLTD). No entanto, o padrão proposto não apresenta padronização nas definições e a maioria das atividades de interpretação requer intervenção humana.

Hafezi, Horri, Shiri e Ghaebi (2013) realizaram uma análise das bibliotecas digitais iranianas, em relação a interoperabilidade. Procuraram identificar a forma de armazenar dados, extrair formatos, protocolos e modelos nas bibliotecas digitais iranianas.

Identificaram que praticamente $80 \%$ das bibliotecas digitais utilizam o formato MARC para descrição bibliográfica, porém, em 45\% delas há alterações no formato. Identificou-se também que $73 \%$ das bibliotecas digitais solicitam que 0 usuário seja membro para que possa ter acesso aos metadados, praticamente inviabilizando as técnicas de coleta de dados.

Nenhuma das bibliotecas digitais apresentam seu esquema de metadados padrão aos usuários e, mais de 70\% tem acesso apenas local, principalmente dentro das próprias redes das bibliotecas físicas. Isso implica em não haver indexação dos objetos digitais por motores de busca públicos. Apenas 9\% do conteúdo está indexado em motores de busca públicos.

Os resultados da pesquisa indicam que $70 \%$ das bibliotecas digitais inviabilizam qualquer processo de interoperabilidade e apenas 30\% indicam possibilidade de interoperabilidade pelo lado do cliente usando o protocolo Z39.50. Apenas uma das bibliotecas planejava usar o protocolo OAl.

Apesar da importância da disseminação de metadados descritivos capazes de suportar a interoperabilidade, a maneira como as instituições estão implementando o Dublin Core na prática é raramente discutida. Refinar a qualidade dos metadados (por exemplo, padronizando o uso de campos como DC: author [Nome], [Sobrenome]) continua a ser um enorme desafio para a interoperabilidade e a harmonização dos perfis para implementações OAI-PMH locais, de forma significativa, apoiaria processos automatizados de colheita (INNOCENTI, VULLO e ROSS, 2010).

0 estado da arte, baseado na literatura consultada, indica claramente que muitos dos papers apresentam diagnósticos de situações problemas em bibliotecas digitais e indicação, após estudos e análises, de soluções com tecnologias e recursos já desenvolvidos e em uso nas comunidades de bibliotecas digitais. Uma parte significativa dos textos indicam problemas sérios no uso de metadados, tanto na questão técnica quanto em sua semântica, inviabilizando processos de interoperabilidade.

Alves (2010, p. 116), afirma que 
0 uso apropriado de um padrão de metadados proporciona uma descrição normalizada e a construção de representações concisas, claras e de qualidade, facilitando o intercâmbio de informações, a interoperabilidade entre sistemas e a recuperação da informação. Para isso, é necessário que seja considerado o tipo de ambiente informacional e, principalmente, qual 0 princípio que norteia o padrão a ser utilizado.

Entende-se, portanto, que seria exaustivo continuar apresentando, nesta seção, outras pesquisas com características muito similares, mudando apenas os objetos e formas de estudo.

\section{A INTEROPERABILIDADE}

0 termo interoperabilidade recebe um conjunto de definições, que podem ser apresentadas de uma forma geral em relação ao termo ou de uma forma específica em relação ao domínio em que se apresenta.

Entre os conceitos mais utilizados de interoperabilidade está o da International Organization for Standardization (ISO) "de que a interoperabilidade é a habilidade de dois ou mais sistemas, que podem ser computadores, meios de comunicação, redes de software e outros componentes de tecnologia de informação, de interagir e de trocar dados a partir de um método definido, objetivando obter os resultados esperados".

Já a National Information Standards Organization (NISO), define interoperabilidade como "a capacidade de sistemas diversos, com diferentes hardwares e plataformas de softwares, estruturas de dados e interfaces, trocarem dados com a mínima perda de conteúdo e funcionalidade" (NISO, 2004, p. 02, tradução nossa).

É fácil notar que os vários conceitos de interoperabilidade, principalmente quando mais genéricos, sintetizam a mesma ideia. Entretanto há definições que já se encaminham para serem mais específicas, tratando do conceito no âmbito dos acervos digitais.

Innocenti, Vullo e Ross (2010) entendem que "A interoperabilidade é uma propriedade referente à capacidade dos sistemas e das organizações de trabalharem em conjunto. Hoje, a interoperabilidade é reconhecida como um passo fundamental na mudança de bibliotecas digitais isoladas para um espaço de informação comum que permitirá aos usuários navegar através de diferentes bibliotecas digitais dentro de um único ambiente integrado."

Conceitua-se nessa pesquisa a interoperabilidade como a capacidade de organizações trocarem informações e dados entre si, independentemente dos sistemas e/ou plataformas que utilizem. Entende-se também a interoperabilidade como aspecto tecnológico fundamental para a evolução e desenvolvimento dos mais variados tipos de acervos digitais, permitindo integração de conteúdo 
de diversas fontes e promovendo a navegação de conteúdos de vários acervos, antes isolados, em ambientes integrados.

Apesar desse texto abordar, majoritariamente, como objeto as questões técnicas de interoperabilidade, sabe-se que não depende apenas de melhores práticas de uso de protocolos, organização e mapeamento de metadados e, alguns outros aspectos técnicos e de conteúdo. De acordo com Candela et al. (2007, p. 55),"a interoperabilidade é uma propriedade multidimensional que se aplica aos recursos de todos os diferentes domínios universais dos acervos digitais, ou seja, conteúdo, funcionalidade, usuário, qualidade, política e arquitetura".

Marcondes e Sayão (2012) apresentam as facetas de interoperabilidade, que são: interoperabilidade técnica, interoperabilidade semântica, interoperabilidade política/humana, interoperabilidade intercomunitária, interoperabilidade legal e interoperabilidade internacional.

Prefere-se adotar nesta pesquisa os níveis de interoperabilidade propostos por Arms (2002), que indica que para haver interoperabilidade são necessários acordos de cooperação em três níveis:

- $\quad 0$ nível técnico: que proporciona a interoperabilidade tecnológica;

- $\quad 0$ nível de conteúdo: que remete a interoperabilidade semântica, na qual a representação e organização do conhecimento são áreas chave a serem estudadas e;

- $\quad 0$ nível organizacional: que se refere à interoperabilidade política, quando organizações se reúnem com o intuito de alcançar a interoperabilidade, implementando padrões e tecnologias que cooperem com este objetivo.

Apesar de entender a importância do nível organizacional da interoperabilidade, onde são tratados elementos como acesso, tipos de pagamento ou licenciamento e autenticação, dar-se-á ênfase às possibilidades de interoperabilidade do ponto de vista técnico, e em alguns momentos apoiado pela questão da interoperabilidade de conteúdo.

\subsection{Categorização da interoperabilidade}

Quando se pensa na integração de bibliotecas digitais e, nas possibilidades de realização de interoperabilidade entre seus dados, é factível que se tente identificar rapidamente quais são os protocolos, os metadados, as formas de acesso, os modelos conceituais, as arquiteturas entre outras características que concebem modelos interoperáveis. 
Tanto do ponto de vista de uso, quanto de proposição de modelos interoperáveis, há sempre dúvidas em quais seriam as características técnicas que poderiam ser utilizadas e como elas funcionam. Identificar as possibilidades de interoperabilidade e entender o funcionamento de todas elas, são as respostas que as perguntas dessa pesquisa pretende responder.

Para tanto apresenta-se aqui um delineamento dessas características que circundam os modelos interoperáveis de acervos digitais.

Para realizar o mapeamento, considera-se que alguns elementos são de fundamental importância, como os protocolos. Em geral, os protocolos utilizam-se de mecanismos, modos de operação ou arquiteturas para realizar os processos de interoperabilidade. Podemos separar os modos de operação dos protocolos em quatro categorias:

\section{a) Agregação}

Na categoria de agregação encontram-se os protocolos que facilitam o processo de exposição e coleta de metadados compartilháveis para que possa ser entendido e usado fora do seu ambiente local. Em geral caracteriza-se por um acervo digital centralizado que realiza a coleta de dados em vários acervos parceiros, constituindo uma interface única e integrada de busca. 0 chamado modelo de harvesting ganhou ênfase em 1999 com o surgimento da Iniciativa dos Arquivos Abertos (Open ArchivesIniciative - OAI). Os protocolos OAI-PMH e OAI-ORE são os mais utilizados nesta categoria.

\section{b) Sindicação (distribuição - servidor-cliente)}

Os protocolos de sindicação, em alguns casos chamados de distribuição, facilitam o fluxo de dados do servidor-cliente através do envio de "feeds" de informações do site para assinantes, o que Ihes permite coletar, organizar e visualizar informações atualizadas com freqüência de várias fontes. Atualmente, RSS e ATOM tornaram-se o padrão de fato no domínio dos protocolos de distribuição.

\section{c) Protocolo publicação}

Essa categoria de interoperabilidade refere-se a capacidade de acervos receberem depósito direto de outros acervos. É o chamado "repository to repository deposit". Nessa categoria ocorre relação de publicação direta de um acervo digital para outro. Esse modo de operação já é bastante 
conhecido em várias plataformas de publicação e gestão de conteúdo. Os protocolos mais utilizados nessa categoria são SWORD e AtomPUB.

\section{d) Busca distribuída (busca federada)}

A categoria de busca distribuída refere-se a capacidade de servidores receberem solicitações de pesquisa federadas e responderem com resultados. Nessa categoria encontram-se os protocolos Z39.50 e seus sucessores (para Web) SRU e SRW.

\section{e) Protocolos}

Os protocolos são um conjunto de regras para definir a comunicação entre sistemas. Aborda-se a seguir os protocolos mais citados nos papers selecionados no processo metodológico desta pequisa:

\section{f) $\mathrm{OAI}-\mathrm{PMH}$}

0 protocolo OAI-PMH introduz o conceito de Metadata Harvesting (colheita de metadados), um processo unilateral, onde, os provedores de serviços (integradores de dados e centrais de pesquisa), a partir da lista de repositórios (provedores de dados), realizam periodicamente uma busca a estes provedores de dados, "colhendo" os metadados para exibição sob a forma de consultas efetuadas pelos usuários.

Projetado para ser genérico, simples e fácil de implementar, o OAI-PMH fornece uma maneira padrão de colher registros de metadados, bem como obter informações básicas e tipos de metadados suportados. O protocolo é baseado em HTTP(Hypertext Transfer Protocol), usando uma URL comum com um parâmetro para selecionar o tipo de solicitação. A troca de mensagens entre o servidor do provedor de dados e 0 programa robô externo do provedor de serviços para a transferência de metadados é unidirecional - 0 provedor de serviços faz solicitações ao provedor de dados, que responde enviando metadados.

As solicitações do provedor de serviço são feitas via protocolo HTTP, usando comandos CGI codificados por meio dos métodos GET ou POST. Os recursos notáveis são: esquemas de metadados extensíveis, mas com o Dublin Core obrigatório para interoperabilidade de nível básico; timestamps nas atualizações de metadados para suportar a colheita incremental apenas do conteúdo alterado; conjuntos hierárquicos para permitir a colheita de subconjuntos definidos; e um esquema de identificador mundial no OAI: URI namespace, para suportar um serviço de resolução central. Um registro OAI é um 
documento XML(eXtensible Markup Language) com um cabeçalho padrão, carga útil de metadados e metadados sobre esses metadados, como informações de direitos.

\section{g) OAI-ORE}

Mais recentemente, o OAI desenvolveu um protocolo para trocar objetos digitais complexos. O OAI-ORE (Object Reuse and Exchange) fornece um mecanismo de interoperabilidade similar para objetos como OAI-PMH fornece para seus metadados. Agregados de modelos ORE, onde um objeto é composto de componentes, cada um dos quais com uma URI. 0 objeto de agregação define a relação entre cada um dos componentes. Um ORE Resource Map contém um objeto de agregação e seus metadados, representados em RDF / XML ou o formato de distribuição de Atom.

Um uso típico de ORE seria representar um objeto composto, como uma impressão composta por vários documentos, para troca de dados com outro repositório. 0 ResourceMap pode diferenciar entre um objeto digital com várias renderizações do mesmo conteúdo e um com vários materiais relacionados combinados. ORE não é um substituto para $\mathrm{PMH}$.

ResourceMaps podem ser transferidos através de solicitações HTTP simples ou um protocolo de transferência em lote, como PMH.

\section{h) $Z 39.50$}

0 Protocolo Z39.50 nasceu de um projeto que envolvia a Online Computer Library Center (OCLC) e a LibrayofCongress (LC) e utiliza-se de um modelo cliente-servidor para possibilitar a busca e recuperação de informações. 0 cliente conecta-se ao servidor, emite uma consulta, que é processada e então recebe os dados oriundos do servidor.

0 protocolo especifica estruturas de dados e regras de intercâmbio que permitem uma máquina cliente pesquisar bancos de dados em uma máquina servidor e recuperar registros que são identificados como resultado de tal pesquisa.

0 modelo arquitetônico básico que o Z39.50 usa é o seguinte: um servidor abriga uma ou mais bases de dados que contenham registros. Associados a cada banco de dados, há um conjunto de pontos de acesso (índices) que podem ser usados para pesquisa. A consulta encontra os pontos de acesso e retorna o resultado. 


\section{i) SIMPLE WEB-SERVICE OFFERING REPOSITORY DEPOSIT (SWORD)}

0 protocolo SWORD foi desenvolvido pela primeira vez em 2007 para abordar a necessidade de uma interface de depósito padronizada para repositórios digitais. 0 protocolo SWORD foi implementado para as plataformas de depósito DSpace, EPrints, Fedora e Intralibrary. O SWORD é baseado no AtomPublishingProtocol (AtomPub), um padrão amplamente adotado nos blogs (e nas plataformas utilizadas para esse fim) e outros sites de conteúdo. 0 AtomPub foi projetado para ser estendido para usos específicos, permitindo que a SWORD forneça extensões para os requisitos colocados pelos repositórios digitais.

SWORD é um padrão de interoperabilidade que permite aos acervos digitais aceitarem o depósito de conteúdo de várias fontes em diferentes formatos, através de um protocolo padronizado. Da mesma forma que o protocolo HTTP permite que qualquer navegador web possa conversar com qualquer servidor web, assim o protocolo SWORD permite aos clientes falar com os servidores de acervos digitais. SWORD foi definido com vistas a oferecer uma solução para facilitar o envio de recursos para sistemas de repositórios acadêmicos.

A comunicação utilizando o SWORD ocorre em duas etapas. A primeira etapa requer que 0 usuário realize a sua autenticação no servidor (acervo). Na segunda etapa é realizado o depósito do objeto digital propriamente dito.

Para a primeira etapa finalizar sem problemas, o usuário deve autenticar-se na plataforma, com direitos de publicar em alguma coleção. Uma vez realizada a autenticação, o objeto digital pode ser enviado ao repositório na segunda etapa, juntamente com seus metadados. Esse objeto será aceito de acordo com a configuração do servidor (tamanho, tipo, metadados).

Em 2011 foi projetada e implantada a segunda versão do protocolo, o SWORD V2. Seu objetivo era ampliar ainda mais o suporte para o AtomPub, apoiando todo o ciclo de vida do depósito. Ele faz isso fornecendo mecanismos para ir além do deposito de recursos, permitindo atualizá-los, substituílos e excluí-los.

\section{j) SEARCH AND RETRIEVE URL (SRU) E SEARH/RETRIEVE WEB SERVICE (SRW)}

Os protocolos SRU e SRW foram concebidos pela LC e propuseram as facilidades e características do protocolo Z39.50 para o contexto mais atualizado da internet, com uso de URL e de serviços web. 
O SRU é um protocolo desenvolvido para trabalhar no âmbito do protocolo HTTP e possibilita a recuperação de registros no formato MARC (Machine Readable Cataloging), este utilizado principalmente por catálogos de bibliotecas, e no formato Dublin Core, o qual é utilizado largamente para a catalogação de arquivos digitais (MOREIRA; RIBEIR0, 2008, p. 171).

O SRU utiliza o serviço Web REST (Representational State Transfer), o qual codifica comandos do cliente para o servidor numa string (sequência de caracteres), na forma de uma URL. Cada um desses valores é especificado no formato nome=valor, e a cada nova especificação é atribuído um novo parâmetro para o servidor.

O SRW foi desenvolvido com o mesmo objetivo do SRU, entretanto utiliza-se de uma arquitetura computacional baseada em Simple Object Access Protocol (SOAP), e não de um URL.

Em termos de estrutura de serviços web, o SRW é identificado como um protocolo baseado em SOAP, enquanto a SRU segue os princípios REST para a entrega de serviços da Web. Apesar da diferença em sua estrutura, a função de ambos os protocolos e seu conjunto de operações suportadas são quase iguais.

\section{k) RDF SITE SUMMARY (RSS)}

0 RSS é um protocolo de distribuição baseado em XML, que é amplamente adotado por provedores de dados atualizados com frequência. Um feed RSS geralmente consiste no título de itens, juntamente com suas apresentações, seguido de links para os registros de texto completo de recursos da Web originais. No entanto, usando módulos, a representação de feeds RSS pode ser estendida para representar formatos de metadados mais descritivos, como Dublin Core através de feeds RSS (BegedDovet al., 2000; Croome, 2002).

Hammond et al. (2004) discutiram novos casos de uso de RSS na publicação acadêmica, como atualizar 0 índice de revistas, alertas de citações, artigos publicados, ETDs enviados e aceitos.

\section{I) ATOM}

0 termo ATOM, no contexto dos protocolos de interoperabilidade, refere-se a dois protocolos relacionados: formato de distribuição de ATOM (ASF), que é um protocolo de distribuição de XML baseado como RSS, utilizado como estrutura de feed da web e ATOM PublishingProtocol (APP), que se refere para um protocolo baseado em HTTP projetado para 
criar, editar e publicar o conteúdo de sites que oferecem suporte a este protocolo. ATOM, de fato, é uma tecnologia paralela ao RSS introduzida para abordar algumas insuficiências de RSS.

Ao contrário do modelo de extensibilidade RSS, o modelo de extensibilidade do ATOM foi projetado de forma a proporcionar interoperabilidade entre as implementações estendidas e as outras implementações que não reconhecem essas extensões.

\subsection{Interoperabilidade do ponto de vista computacional}

Do ponto de vista computacional a interoperabilidade apresenta-se com muitas variáveis e requisitos, que vão desde a estrutura de arquivo a ser trocado até os modelos funcionais de acesso computacional.

Do ponto de vista de estrutura de arquivos, é fácil notar que a grande maioria dos protocolos já apresentados utiliza-se da linguagem XML para troca de informações. Com exceção do protocolo Z39.50, que se apoia em um modelo baseado em arquivos binários, todos os outros protocolos tem no XML sua estrutura padrão para troca de arquivos. Alguns protocolos já evoluem e permitem formatos serializados mais novos em relação ao XML, caso do JSON (JavaScriptObjectNotation), como faz 0 SRW.

0 modelo JSON, vem substituindo o XML como estrutura de arquivo para troca de dados. Por facilitar a leitura e escrita por humanos e interpretação e geração por máquinas, este subconjunto da linguagem de programação JavaScript usa convenções que são familiares às linguagens C, Java, JavaScript, Perl, Python e muitas outras. Estas propriedades fazem com que JSON seja um formato ideal de troca de dados.

JSON está constituído em duas estruturas:

- $\quad$ Uma coleção de pares nome/valor. Em várias linguagens, isto é caracterizado como um object, record, struct, dicionário, hashtable, keyedlist, ou arrays associativas.

- Uma lista ordenada de valores. Na maioria das linguagens, isto é caracterizado como uma array, vetor, lista ou sequência.

Pensando no modelo de comunicação computacional, os protocolos RSS e Atom utilizam-se de um padrão que a informação parte do servidor para o cliente, entretanto todos os outros protocolos 
são baseados em conexões do tipo cliente/servidor, onde há uma conexão (autenticada ou não) por parte do equipamento cliente para solicitar informações do servidor, que responde de acordo com as solicitações recebidas.

Destacam-se alguns modelos funcionais de comunicação computacional como os WebServices e 0 uso de APIs.

\section{a) WEB SERVICES}

Um Web Service é um sistema de software, identificado através de uma URI, na qual interfaces públicas e modos de conexão são definidos e descritos em XML. Estas definições podem ser descobertas por outros sistemas de software. Estes sistemas podem então interagir com o Web Service em uma maneira prescrita pela sua definição, usando mensagens baseadas em XML e transportadas por protolocos da Internet (WSA 2003).

Entre as abordagens existentes para a implementação de Web Services, os protocolos SOAP e o REST são as opções de maior destaque e uso, estando presentes em grande parte das discussões relacionadas a arquiteturas orientadas a serviços na web.

0 protocolo SOAP teve grande importância a partir de 2003, quando passou a ser uma recomendação da W3C para desenvolvimento de serviços web, sendo o padrão mais implementado na época e deixando um legado de sistemas e integrações que perdura até hoje.

O SOAP é um protocolo para troca de informações estruturadas em plataformas descentralizadas e distribuídas, utilizando tecnologias baseadas em XML.

0 uso do SOAP tem como vantagem a sua independência em relação às linguagens de programação, a sua simplicidade e o fato de ser extensível, o que permite a sua utilização em qualquer aplicação. Pode-se citar ainda como vantagem o fato do SOAP poder realizar as suas chamadas sobre 0 protocolo HTTP e ser estruturado em XML, ou seja, duas tecnologias instituídas como padrão, neutras, e usadas em larga escala.

0 REST, por sua vez, foi desenvolvido juntamente com o protocolo HTTP $1.1 \mathrm{e}$, ao contrário do SOAP, que tem como objetivo estabelecer um protocolo para comunicação de objetos e serviços, propôs algumas ideias de como utilizar corretamente os verbos HTTP (GET, POST, PUT, HEAD, OPTIONS e DELETE) para criar serviços que poderiam ser acessados por qualquer tipo de sistema. 
Sua concepção, portanto, não era de um protocolo, mas sim de um Design Pattern arquitetural para serviços expostos numa rede, como a internet, através do protocolo HTTP.

0 uso de WebServices para interoperabilidade garante um conjunto de vantagens às plataformas de acervos digitais, dentre elas: uma interface abstrata para acesso aos métodos disponibilizados, ocultando detalhes de implementação do usuário do serviço; sendo baseado em um padrão aberto, estruturado em XML, garante-se a portabilidade das mensagens mesmo sob diferentes plataformas de operação; é possível garantir mais segurança com criptografia das informações trafegadas; e um dos principais benefícios, a economia de recursos, pois não consomem recursos de comunicação enquanto em estado de espera.

\section{b) API (APPLICATION PROGRAMMING INTERFACE)}

API é um conjunto de rotinas e padrões de programação para acesso a um aplicativo de software ou plataforma baseado na Web. A sigla API refere-se ao termo em inglês "Application Programming Interface" que significa em tradução para o português "Interface de Programação de Aplicativos".

Uma API é criada quando uma organização/plataforma tem a intenção de que outros criadores de software desenvolvam produtos e serviços associados ao seu serviço. A ideia principal das APIs é que parte das funcionalidades de um serviço possam ser usadas em outras plataformas da maneira mais assertiva e conveniente para seus usuários.

Através das APIs, os aplicativos podem se comunicar uns com os outros sem conhecimento ou intervenção dos usuários. Elas funcionam através da comunicação de diversos códigos, definindo comportamentos específicos de determinado objeto em uma interface. A API liga as diversas funções em um site de maneira que possam ser utilizadas em outras aplicações. De modo geral, a API é composta de uma série de funções acessíveis somente por meio de programação.

As APIs normalmente funcionam integradas ou são parte das funcionalidades de um Web Service.

Grande parte das plataformas disponíveis para implementação de acervos digitais como Dspace, Eprints e Fedora, além de iniciativas como Europeana e DPLA disponibilizam acesso ao seu conteúdo por meio de APIs, permitindo inclusive, com essa tecnologia, que possa ser feita integração direta de seus dados com outros acervos digitais. 


\subsection{Mapeamento da interoperabilidade}

Apresentar os protocolos, sua divisão em categorias e as estruturas computacionais relativas a interoperabilidade, permitiu constituir um mapeamento dos protocolos mais utilizados em plataformas que implementam acervos digitais.

Por meio da Figura 1 é possível identificar os modelos de interoperabilidade - apresentados aqui como as categorias em que se dividem os protocolos - e também a estrutura computacional de operação utilizados pelos principais protocolos identificados para interoperabilidade técnica em integração de acervos digitais. As categorias estão apresentadas como colunas e a estrutura computacional como linhas na Figura 1.

Figura 1 - Mapeamento da interoperabilidade técnica

\begin{tabular}{|c|c|c|c|c|}
\hline $\begin{array}{c}\text { Busca Distribuída } \\
\text { SRW }\end{array}$ & $\begin{array}{l}\text { Protocolo de } \\
\text { Publicação }\end{array}$ & Agregação & Sindicação & WebService - SOAP \\
\hline SRU & $\begin{array}{l}\text { AtomPub } \\
\text { SWORD }\end{array}$ & $\begin{array}{l}\text { OAI-PMH } \\
\text { OAI-ORE }\end{array}$ & & WebService - Rest \\
\hline \multirow[t]{2}{*}{ z39.50 } & & & & Cliente -> Servidor \\
\hline & & & $\begin{array}{l}\text { RSS } \\
\text { Atom }\end{array}$ & Servidor $->$ Cliente \\
\hline
\end{tabular}

Fonte: autores

0 mapeamento apresentado tem como objetivo favorecer a identificação, uso e implementação dos protocolos nos processos de interoperabilidade entre acervos digitais.

\subsection{Interoperabilidade semântica}

As questões semânticas relacionadas a interoperabilidade estão enquadradas no nível de conteúdo, dos níveis de interoperabilidade de Arms (2002). Neste contexto entende-se que implementar interoperabilidade semântica está diretamente relacionado a entender e associar conceitos (mesmo que diferentes) dos dois lados que se relacionarão quando ocorrer o processo de interoperabilidade. 
A semântica, assim como a interoperabilidade de conteúdo está diretamente relacionada ao uso de metadados, ou padrões de metadados.

Alguns protocolos, como o OAI-PMH, primam pelo uso do padrão de metadados Dublin Core, entretanto mesmo esse padrão sendo obrigatório no protocolo é possível expandir o uso do OAIPMH com a extensão do DublinCore ou uso de outro padrão de metadados. Apesar do Dublin Core ser o padrão de metadados mais utilizados nas mais variadas plataformas que implementam acervos digitais, ele tem sido substituído por padrões mais adequados para uso em domínios mais específicos, em alguns casos sendo substituídos por perfis de aplicação também conhecidos como application profiles, discutidos a seguir.

Quando se troca dados com padrões de metadados já previamente estabelecidos, não deveria haver dúvida sobre a semântica dos elementos, entretanto em alguns casos os padrões de metadados entre as plataformas é distinto, sendo necessário realizar uma tarefa chamada de mapeamento ou crosswalk.

0 Crosswalk consiste em realizar um mapeamento dos elementos entre dois padrões de metadados distintos, esse processo é normalmente norteado pelo conhecimento de cada um dos elementos dos dois padrões e pela associação entre pares de elementos dos dois padrões.

0 impacto que as tecnologias da Web Semântica vêm apresentando em relação a todo contexto da internet, mas principalmente sobre acervos digitais, tem favorecido o uso do padrão RDF para dar significado a cada ligação efetuada no mapeamento (crosswalk) de padrões de metadados.

0 domínio e entendimento do padrão RDF também trouxe uma nova concepção de uso aos padrões de metadados, os perfis de aplicação, que são padrões que passaram a ser constituídos para suprir a carência técnica e funcional de alguns padrões de metadados tradicionalmente utilizados.

Perfil de aplicação é um conjunto de elementos de metadados selecionados de um ou mais esquemas de metadados, combinados em um esquema composto. Os perfis de aplicação oferecem meios para se expressar os princípios de modularidade e extensibilidade. Sua finalidade é combinar esquemas de metadados adaptando-os às exigências funcionais de aplicações específicas, e mantendo a interoperabilidade com esquemas originais que serviram como base. Seu maior objetivo é facilitar a interoperabilidade semântica (DUVAL et al., 2002). Os perfis de aplicação são constituídos para atender a demandas específicas dentro de uma área ou domínio de aplicação. 
São exemplos de perfis de aplicação o AGRIS (Agricultura Network) para a área da agricultura, 0 SWAP (Scholary Works Application Profile) para a ciência, o EDM (Europeana Data Model) da Europeana para questões de patrimônio cultural, entre muitos outros.

Não há dúvidas que o uso de perfis de aplicação em substituição aos tradicionais padrões de metadados dificultou os processos de interoperabilidade.

Uma nova tendência identificada nos modelos de interoperabilidade semântica é o que tem se chamado atualmente de meta-modelos (ROLAN, 2017). Os meta-modelos visam não apenas mapear metadados para encontrar possíveis correspondências entre eles, mas atuam buscando um nível ontológico, o mais genérico possível para um domínio, integrando diferentes esquemas de metadados a partir de uma ontologia de alto nível que represente as conexões possíveis e desejadas entre os diferentes modelos.

Há ainda uma nova iniciativa, chamada de IIIF (Internation Image Interoperability Framework), que é constituída por um conjunto de interfaces de aplicativos de programação (APIs) baseados em padrões abertos da Web para interoperabilidade de imagens.

Por meio do IIIF, instituições com plataformas diferentes, mesmo que com servidores de imagens diferentes, podem compartilhar e reutilizar o conteúdo umas das outras, através de um conjunto de técnicas computacionais.

Segundo Fielding e Taylor (2000) a arquitetura lógica das APIs IIIF baseia-se nas especificações REST para recuperar páginas da Web e identificar adequadamente os recursos com URIs (UniformResourceldentifier), permitindo que os servidores forneçam dados estruturados aos clientes em formato de padrão aberto, como HTML (HyperTextMarkupLanguage), XML ou JSON (SALARELLI, 2017).

A utilização das APIs IIIF para as imagens por vários repositórios permitirá 0 seu acesso simultâneo através de uma única interface, ou seja, possibilitará a interoperabilidade entre servidores e clientes.

Pensando em modelos semânticos, do ponto de vista técnico, a linguagem JSON-LD (JSON-Linked Data) tem se tornado o modelo de serialização favorito para troca de dados, pois trata-se de um modelo de vinculação de dados usando JSON. O JSON-LD funciona com o conceito de "contexto" que possibilita construir mapeamentos adicionais ao JSON baseado no modelo RDF. 0 contexto vincula propriedades do objeto em um documento JSON para conceitos em uma ontologia. 
Ainda sobre a questão de conteúdo, é importante registrar que muitas plataformas constituem seus próprios modelos e formatos de objetos digitais, encapsulados em modelos proprietários, para interoperabilidade apenas entre acervos que utilizam a mesma plataforma.

\section{CONSIDERAÇÕES FINAIS}

Essa pesquisa procurou refletir acerca das várias possibilidades de interoperabilidade disponíveis para integração de acervos digitais. Notou-se, claramente, no levantamento bibliográfico que os papers mais atuais cobrem principalmente questões relacionadas a problemas de interoperabilidade e sugestões de uso de padrões e técnicas já conhecidas na área. Verificou-se que muitos dos problemas de interoperabilidade apresentados estão na má utilização dos padrões de metadados, ou ainda na ausência deles na implementação dos acervos digitais.

Considera-se que a má utilização dos padrões é um problema muito recorrente mas que poderia ser evitado. Entretanto denotou-se também no referencial pesquisado que não há exploração máxima dos recursos de interoperabilidade que as principais plataformas disponibilizam.

Dessa forma essa pesquisa buscou apresentar os vários protocolos utilizados para favorecer os processos de interoperabilidade entre plataformas que implementam acervos digitais, de maneira que possam ser utilizados de forma mais consistente por equipes que pretendem integrar dados de acervos distintos, mas também para impulsionar a implementação dos mesmos em novas plataformas que possam ser criadas.

Iniciou-se apresentando as categorias dos protocolos, com destaque para sindicação (distribuição), agregação, busca distribuída e protocolo de publicação, e na sequência apresentou-se os protocolos com suas características.

Foi possível verificar que grande parte dos protocolos utiliza-se da estrutura computacional de Web Services para realizar a troca de dados e que a estrutura fundamental dos arquivos trocados é baseada na linguagem XML. A partir destas informações constituiu-se um mapa de interoperabilidade para uso de equipes que pretendem realizar processos de interoperabilidade entre acervos digitais.

Verificou-se ainda que a questão técnica da interoperabilidade, apesar de discutida e rediscutida em muitos papers e pesquisas científicas, ainda apresenta-se como dificultador nos processos de troca 
de informações e agregação de acervos digitais, posicionando-se com um dos principais entraves para a integração de dados.

Apesar de questões políticas e organizacionais ainda serem de fundamental impacto para que acervos digitais possam trocar dados, os resultados da pesquisa bibliográfica mostram que os problemas ainda permanecem na interoperabilidade técnica e de conteúdo.

Notou-se, entretanto, que grande parte dos acervos digitais foram criados (idealizados) para serem únicos, e somente nos últimos anos tem se pensado em integrar dados já na idealização dos acervos digitais, o que pode ser um alento, visto que mais recentemente a filosofia de construção de novos acervos digitais já embutem na sua origem o ideal de um dia serem interoperáveis.

Observou-se novas tendências, como o uso de meta-modelos que podem facilitar a convivência entre diferentes esquemas de metadados em novos níveis de agregação ontológica, facilitando lidar com a inerente diversidade e complexidade dos diferentes modelos atualmente existentes e que terão de ser interconectados de alguma maneira em novos projetos, que terão o desafio de lidar com dados e sistemas legados.

Pretende-se aprofundar, em estudos futuros, na avaliação de meta-modelos atualmente em uso e seu potencial para implementação em produtos e serviços de informação.

\section{REFERÊNCIAS}

ALVES, R. C. V. Metadados como elementos do processo de catalogação. 2010. 134f. Tese (Doutorado em Ciência da Informação) - Faculdade de Filosofia e Ciências, Universidade Estadual Paulista, Marília, 2010.

BEGED-DOV, G.et al. RDF site summary 1.0 modules. Dublin Core. 2000.

CANDELA, L., et al. DELOS network of excellence on digital libraries. The DELOS Digital Library ReferenceModel (version 0.98). n. 55, Nov. 2007. Disponível em: http://www.delos.info/files/pdf/ ReferenceModel/DELOS_DLReferenceModel_096.pdf>. Acesso em: 10 nov. 2017.

CROOME, C. RDF site summary 1.0 modules.Qualified Dublin Core. 2002.

DUVAL, E. et al. Metadata principles andpracticalities. Disponível em: http://www.dlib.org/dlib/ april02/weibel/04weibel.html. Acessoem: 12 nov.2017. 
FIELDING, R. T.; TAYLOR, R. N. Principled design of the modern web architecture. In: INTERNATIONAL CONFERENCE ON SOFTWARE ENGINEERING (ICSE '00), 22., 2000, New York. Proceedings... ACM: New York. p. 407-416. Disponível em: https://www.ics.uci.edu/ fielding/pubs/ webarch_icse2000.pdf. Acesso em: 15 set. 2017.

FUSCO, E. Modelos conceituais de dados como parte do processo da catalogação: perspectiva de uso dos FRBR no desenvolvimento de catálogos bibliográficos digitais. Tese (Doutorado em Ciência da Informação). Faculdade de Filosofia e Ciências, Universidade Estadual Paulista "Júlio de Mesquita Filho", Marília, 2010. Disponível em: http://www.marilia.unesp.br/Home/Pos-Graduacao/ Cienciadalnformacao/Disser tacoes /fusco_e_do_mar.pdf. Acesso em: 12 nov. 2017.

HAFEZI, M. A.et al. Digital library interoperability: proposing a model. International Journal of Information Science and Management, v. 3, n. 1, p. 57 - 75, Jan./Jun. 2013.

HAMMOND, T., HANNAY, T.; LUND, B. The role of RSS in science publishing. D-Lib Magazine, v. 10, n. 12, 2004.

INNOCENTI, P.; VULLO,G.; ROSS, S. Towards a digital library policy and quality interoperability framework: the dl.org project. New Review of Information Networking, v.15, p.29-53, 2010.

MARCONDES, C. H.; SAYÃO L. F. Integração e interoperabilidade no acesso a recursos informacionais eletrônicos em C\&T: a proposta da Biblioteca Digital Brasileira. Ciência da Informação, Brasília, v. 30, n. 3, p. 24-33, 2001. Disponível em: http://www.ibict.br/. Acesso em: 17 nov. 2017.

MARTINS, D. L.; SILVA, M. F. Critérios de avaliação para sistemas de bibliotecas digitais: uma proposta de novas dimensões analíticas. InCID: Revista de Ciência da Informação e Documentação, Ribeirão Preto, v. 8, n. 1, p. 100 - 121, abr. 2017. Disponível em: https://www.revistas.usp.br/incid/ article/view/. Acesso em: 25 jan. 2018.

MENZIES, K.; BIRRELL, D.; DUNSIRE, G.An investigation of information systems interoperability in UK universities: findingsand recommendations. New Review of Information Networking, v.16, p. 92-140, 2011.

MOREIRA, W.; RIBEIRO, T. Introdução ao uso dos protocolos SRU/SRW: ferramentas para a catalogação cooperativa. Perspectivas em Ciência da Informação, v.13, n.3, p.167-182, set./dez. 2008. MORGAN, E. L. An Introduction to the Search/Retrieve URL Service (SRU). 2004. Disponível em: http://www.ariadne.ac.uk/issue40/morgan/intro.html. Acessoem: 19 nov. 2017.

POTVIN, S.; THOMPSON, S. An analysis of evolving metadata influences, standards, and practices in electronic theses and dissertations. LRTS, v.60, n. 2, p. 99 -114. 2017.

ROLAN, G. Towards interoperable recordkeeping systems: a meta-model for recordkeeping metadata. Records Management Journal, v. 27, n. 2, p.125-148, 2017. Disponível em: https://doi.org/10.1108/ RMJ-09-2016-0027. Acessoem: 10 de nov. 2017. 
ROSS, S. Digital library development review. Final report. National Library of New Zealand, 2003. Disponívelem: http://eprints.erpanet.org/50/01/ross_ report.pdf. Acesso em: 30 out. 2017.

SALARELLI, A. InternationallmageInteroperability Framework (IIIF): unapanoramica. JLIS.it, Firenze, v. 8, n. 1, Jan. 2017, p. 5-66. Disponível em: https://www.jlis.it/article/view/12090/11177. Acesso em: 10 fev. 2017.

WEB Services Architecture, 2003. Disponível em: http://www.w3.org/TR/2002/WD-wsarch-20021114/. Acesso em: 20 out. 2017. 